\title{
MAC protocol with grouping awareness GMAC for large scale Internet-of-Things network
} \author{
Allehaibi $^{2}$, Nasser Albogami $^{2}$, Anton Satria Prabuwono ${ }^{2}$ \\ ${ }^{1}$ Center for Cyber Security, Universiti Kebangsaan Malaysia UKM, Bangi, Selangor, Malaysia \\ 2 Department of Computer Science, King Abdulaziz University, Jeddah, Saudi Arabia \\ Corresponding Author: Rosilah Hassan \\ Email address: rosilah@ukm.edu.my
}

Abdulrahman Sameer Sadeq ${ }^{1}$, Rosilah Hassan ${ }^{\text {Corresp., }}{ }^{1}$, Azana Hafizah Mohd Aman ${ }^{1}$, Hasimi Sallehudin ${ }^{1}$, Khalid

The development of Medium Access Control (MAC) protocols for Internet of Things should consider various aspects such as energy saving, scalability for a wide number of nodes, and grouping awareness. Although numerous protocols consider these aspects in the limited view of handling the medium access, the proposed Grouping MAC (GMAC) exploits prior knowledge of geographic node distribution in the environment and their priority levels. Such awareness enables GMAC to significantly reduce the number of collisions and prolong the network lifetime. GMAC is developed on the basis of five cycles that manage data transmission between sensors and cluster head and between cluster head and sink. These two stages of communication increase the efficiency of energy consumption for transmitting packets. In addition, GMAC contains slot decomposition and assignment based on node priority, and, therefore, is a grouping-aware protocol. Compared with standard benchmarks IEEE 802.15.4 and industrial automation standard 100.11a and userdefined grouping, GMAC protocols generate a Packet Delivery Ratio (PDR) higher than $90 \%$, whereas the PDR of benchmark is as low as $75 \%$ in some scenarios and $30 \%$ in others. In addition, the GMAC accomplishes lower end-to-end (e2e) delay than the least e2e delay of IEEE with a difference of $3 \mathrm{~s}$. Regarding energy consumption, the consumed energy is $28.1 \mathrm{~W} / \mathrm{h}$ for GMAC-IEEE Energy Aware (EA) and GMAC-IEEE, which is less than that for IEEE 802.15.4 (578 W/h) in certain scenarios. 


\title{
2 MAC Protocol with Grouping Awareness GMAC for
}

3 Large Scale Internet of Things Network

4

\author{
Abdulrahman Sameer Sadeq ${ }^{1}$, Rosilah Hassan ${ }^{1}$, Azana Hafizah Mohd Aman ${ }^{1}$, Hasimi \\ Sallehudin ${ }^{1}$, Khalid Allehaibi ${ }^{2}$, Nasser Albogami ${ }^{2}$, Anton Satria Prabuwono ${ }^{2}$ \\ ${ }^{1}$ Center for Cyber Security, Faculty of Information Science and Technology, Universiti \\ Kebangsaan Malaysia, 43600 UKM, Bangi, Selangor, Malaysia \\ ${ }^{2}$ Department of Computer Science, Faculty of Computing and Information Technology, King \\ Abdulaziz University, Jeddah 21589, Saudi Arabia
}

Corresponding Author:

Rosilah Hassan ${ }^{1}$

Email address: rosilah@ukm.edu.my

\section{Abstract}

The development of Medium Access Control (MAC) protocols for Internet of Things (IoT) should consider various aspects such as energy saving, scalability for a wide number of nodes, and grouping awareness. Although numerous protocols consider these aspects in the limited view of handling the medium access, the proposed Grouping MAC (GMAC) exploits prior knowledge of geographic node distribution in the environment and their priority levels. Such awareness enables GMAC to significantly reduce the number of collisions and prolong the network lifetime. GMAC is developed on the basis of five cycles that manage data transmission between sensors and cluster head and between cluster head and sink. These two stages of communication increase the efficiency of energy consumption for transmitting packets. In addition, GMAC contains slot decomposition and assignment based on node priority, and, therefore, is a grouping-aware protocol. Compared with standard benchmarks IEEE 802.15.4 and industrial automation standard 100.11a and user-defined grouping, GMAC protocols generate a Packet Delivery Ratio (PDR) higher than 90\%, whereas the PDR of benchmark is as low as $75 \%$ in some scenarios and $30 \%$ in others. In addition, the GMAC accomplishes lower end-to-end (e2e) delay than the least e2e delay of IEEE with a difference of 3s. Regarding energy consumption, the consumed energy is $28.1 \mathrm{~W} / \mathrm{h}$ for GMAC-IEEE Energy Aware (EA) and GMAC-IEEE, which is less than that for IEEE $802.15 .4(578 \mathrm{~W} / \mathrm{h})$ in certain scenarios.

\section{Introduction}


39

40

41

42

43

44

45

46

47

48

49

50

51

52

53

54

55

56

57

58

59

60

61

62

63

64

65

66

67

68

69

70

71

72

73

74

75

76

77

78

The recent development of Wireless Sensor Networks (WSNs) and the incorporation of technologies of Internet of Things (IoT) has enabled their applications in various industrial fields, particularly through IoT-based WSN (IoT-WSN) (Hassan et al., 2020). Such the emergence has led to numerous applications in different sectors such as agriculture (Hassan, 2020; Keswani et al., 2018), smart cities (Al-Majhad et al., 2018; Nassar et al. 2019; Zhang, 2020), intelligent transportation system (Muthuramalingam et al., 2019), medical field (Onasanya \& Elshakankiri, 2019; Yao et al., 2019), security and surveillance (Benzerbadj et al., 2018; Memon et al., 2020), military (Zieliski, Chudzikiewicz \& Furtak, 2019), forensics (Yaqoob et al., 2019), education, and voting (Srikrishnaswetha, Kumar \& Mahmood, 2019). Sensing-based applications that monitor and gather data are regarded as common applications of IoT (Sadeq, 2018; Wu, Wu \& Yuce, 2018). Figure 1 shows a conceptual diagram of a healthcare monitoring application using IoT-WSN. Body area networks are installed on patients in hospitals, and they continuously gather data from all patients in real time. The sensors are deployed in 3D based configuration, and the sensors are located in each patients' room separately from other patients' rooms, which emphasize the assumption of clusters-based decomposition. The collected data are then used within an intelligent system to assign care algorithmically to increases the recovery ratio. For one floor, one sink connects the clusters, each representing one patient with sensors on different parts of the body. On the other end, the sink is connected to a Software Defined Network (SDN) controller connected to an application layer in the cloud to monitor patients and assign tasks to doctors.

This application requires continuous sensor data collection and transfer to the cloud. The wireless nature of the network and the limited resources of its nodes create two issues. First, the management of node access to the medium must be coordinated with consideration of the sensing rate, sensors' nature, and their relation to the application. This issue affects Quality of Service (QoS) metrics in the network. Second, the management of energy in the network affects the lifetime metric. These issues are not independent of each other, enabling Carrier Sense Multiple Access/Collision Avoidance (CSMA/CA) mode may cause failure in several sensors in accessing the medium, which eventually leads to energy waste and shorter lifetime. However, saving the node energy requires careful scheduling and management of their access to the medium.

Numerous types of Medium Access Control (MAC) protocols are available. A few of the most widely used are IEEE 802.15.4 and ISA 100.11a as IoT MAC protocols in numerous types of applications. These types have small differences in terms of enabling or ignoring packet life time or adding priority to packets or not. One critical issue of these two protocols is the scalable energy awareness solution (Sotenga, Djouani \& Kurien, 2020). When an application requires installing a high number of sensor nodes, the protocol may be inefficient in terms of energysaving due to the resulting collisions. Another issue is the non-awareness of various priority and grouping aspects of the sensor nodes. Such an awareness is important to manage the medium effectively. Recent variants are developed with grouping awareness, such as User-Defined Grouping (UDG) (Yasari et al., 2017) based on ISA 100.11a. However, this variant has various 
79 limitations and necessitates developing of IoT-oriented MAC protocol with scalability, energy

80 efficiency, and grouping awareness. The article aims to propose a novel protocol based on both

81 IEEE 802.15.4 and ISA 100.11a to improve their CAP performance based on exploiting prior

82 knowledge about the clustering information of the network and the priority level of the nodes.

83 Therefore, this study aims to develop two novel variants of Grouping MAC (GMAC) based on

84 current benchmarks, namely, GMAC-IEEE and GMAC-ISA.

85

86

87

88

89

90

91

92

93

94

95

96

97

98

99

100

101

102

103

104

105

106

107

108

109

110

111

\section{Literature Review}

In addition, several improvements on IEEE 802.15.4 in various aspects have been performed, such as the Clear Channel Assessment (CCA) and its effect on the delay and overhead on the protocol. An improvement on CCA (Wang, Liu \& Yin, 2018) is proposed using a graded tailoring strategy, which checks the length of the original packet and modifies its original size according to the partition points. Assuming that the same back-off unit size is used, the protocol includes 20 symbols. To make the data packet tail size 8 , those with lower than 8 add zeroes, and those higher than 8 subtract zeroes. This improvement is useful from the general perspective of delay and over-head, but it ignores prior knowledge of nodes or packets priorities. The mechanism of CCA of IEEE 802.15.4 is also examined in different ways. For example, the CCA has been modified to include primary and secondary stages (Gamal et al., 2020). In addition, an optimization model is built for the delay with energy consumption as a constraint. The model is solved using linear quadratic programming, but it does not consider retransmission essential in IEEE 802.15.4.

Another modification to IEEE 802.15.4 (Patel \& Kumar, 2017) aims to increase the number of CCA from one to two, reducing the number of back-off periods to confirm the status decision of the channel and scarify the low energy consumption of CCA. This modification avoids highenergy consumption when a failure occurs and bandwidth loss if the channel becomes idle. The number of retransmissions and their effect on performance is also examined. The network nodes are divided into sub-groups or classes according to the number of failed retransmission (Henna \& Sarwar, 2018). Specifically, the low number of failed retransmissions implies low increases in the back-off time and converts the protocol of IEEE 802.15.4 from a fixed to an adaptive back-off. However, the approach lacks an automatic means to decide to change the back-off for each class. Another issue is the neglect of energy level of each sensor that is considered a highly critical aspect in the performance. Furthermore, the approach does not embed prior knowledge regarding the sensor's class or priority related to its function in the system.

TDMA and CSMA functionalities of IEEE 802.15.4 are also combined for WSN scheduling with the support of demand, that is, profile. A proposed WSN scheduling based on the concept of network virtualization (Uchiteleva, Shami \& Refaey, 2017) divides the networks into profiles, each

115 of which indicates a set of nodes sharing the same channel demand nature or characteristics. The

116 scheduling proposes two profile categories, bursty and periodic. The super-frame in IEEE802.15.4

117 is then decomposed into contention access frame and contention free frame (Uchiteleva et al., 118 2017), which contains a set of guaranteed time slot. Next, an optimization is conducted to 
119 maximize the utility for each profile. The algorithm uses a greedy optimization approach.

120 Furthermore, as stated in (Shrestha, Hossain \& Choi, 2014), the strength of CSMA/CA when it is

121 combined with TDMA improves the scalability by preserving the performance of legacy-based

122 CSMA/CA-based MAC scheme in congested networks.

123 Other approaches in the literature aim to improve ISA $100.11 \mathrm{a}$, which is regarded as a common

124

125

126

127

128

129

130

131

132

133

134

135

136

137

138

139

140

141

142

143

144

145

146

147

148

149

150

151

152

153

154

155

156

157

158 protocol for industrial wireless sensor networks owing to its wide use in MAC layer management of sensors of control systems (Florencio, Doria Neto \& Martins, 2020). In a recent survey (Raptis, Passarella \& Conti, 2020), a comparison between ISA 100.11a and WirelessHART has been conducted to conclude the need to optimize various aspects in ISA such as communication and energy optimization. An optimization of ISA under TDMA (Satrya \& Shin, 2020) proposes a solution representation that provides a code for each node according to its time slot. Next, the work of (Yasari et al., 2017) develops a genetic-based scheduling algorithm that enables flexible scheduling in ISA 100.11a. However, this work only optimizes one parameter in ISA 100.11a, the packet lifetime assigned to each group, and another parameter outside ISA 100.11a, which is the number of nodes in each group. An objective function is then used to maximize the number of nodes and the distribution of nodes in the groups according to their weights. In addition, this work ignores a direct optimization to the network performance measures such as QoS, which is used as constraint in the optimization only. Some researchers aimed to enhance ISA 100.11a in the context of application, such as adapting ISA to operate in a specific control environment. In (Herrmann \& Messier, 2018), an optimization of the scheduling and the routing (cross-layer) is proposed. The goal is to minimize energy consumption and prolong the lifetime of the petroleum refinery process. The frame structure and an optimization of the scheduling and selection of the routing hops, are the elements of ISA enhancement. Despite the many developments of ISA 100.11a and IEEE 802.15.4 and other related WSN scheduling, some researchers proposed different improvement perspectives. For example, in (Farayev et al., 2020), the pre-knowledge of the periodic nature of data generated in the network is exploited to formulate joint optimization of scheduling, power control, and rate adaptation for discrete rate transmission mode. Although this assumption is useful when it is valid, many WSNs have no pre-knowledge of the nature of data generation, such as event-based monitoring.

The literature on MAC layer scheduling in IEEE 802.15.4 and ISA 100.11a tackles various aspects of these two protocols. Several approaches focus on optimizing CSMA (Gamal et al., 2020) and others pay attention to TDMA (Osamy, El-Sawy \& Khedr, 2019), but limited work concentrates on integrating both functionalities (Wang et al., 2018). Furthermore, none of the previous work develops protocols for integrated CSMA-TDMA with grouping awareness. This factor affects the performance that relies heavily on prior knowledge about each node in terms of its application or role in the system or its priority of responding when packets are generated, compared with systems that consider scheduling of medium access but not the source or group of node priority.

Overall, the handling of the problem of MAC scheduling in IEEE 802.15.4 based protocols, despite its covering to various development aspects such as optimization of parameters, 
159

160

161

162

163

164

165

166

167

168

169

170

171

172

173

174

175

176

177

178

179

180

181

182

183

184

185

186

187

188

189

190

191

192

193

194

195

196

incorporation of adaptive approaches, usage of TDMA and/or CMSA, non-of the previous approaches has addressed the scheduling with a consideration of the node's geographical distributions. Considering that the collisions occur more frequently when the nodes are close to each other or share the same coverage zone, relative location-aware contention is an important criterion for optimizing the scheduling and reducing the collisions. The article aims to propose a novel MAC scheduling protocol based on IEEE 802.15.4 that enables clustering, which is location-based grouping as a criterion for handling scheduling. Furthermore, the proposed protocol will exploit TDMA to exchange clusters' information with the sink and CMSA for transmitting information within the clusters to the cluster heads. With such scheduling management, the developed protocol is the first MAC scheduling that jointly enables clustering awareness and CSMA-TDMA integration in a single protocol.

\section{Materials \& Methods}

This section provides the developed protocol and the evaluation methods and metrics used to compare with state-of-the-art protocols or benchmarks. We divide the methodology into several parts. At first, we present the assumptions and symbols, and the network hierarchy. Next, the energy model and the energy-aware back-off time along with the protocol design are provided. In the protocol design, we provide the network cycle and the protocol activity diagram.

\section{Assumptions and Symbols}

We assume that the network is represented by a graph $G=(V, E)$ with $V=\left\{n_{1}, n_{2}, \ldots n_{K}\right\}$, where $n_{i}$ denotes the sensor $i$, and $\mathrm{k}$ denotes the number of sensors. Each sensor $i$ is located in position $\left(x_{i}, y_{i}, z_{i}\right) \in R^{3} . E$ denotes the set of links between the nodes.

i. The clustering information, the number of groups in the network, and the priorities of the nodes are defined in advance based on the application.

ii. The network consists of non-overlapping clusters in the coverage of sensor nodes when using low power transmission mode, but cluster heads connect to the sink when using highpower transmission mode.

iii. The network consists of several priority groups $L_{j}=1,2$. maxGroup, where maxGroup denotes the maximum number of groups of sensors, and $\boldsymbol{j}$ denotes an index of the priority level. Lower $\mathrm{j}$ is equivalent to higher priority according to Equation 6.

iv. Each node creates an array with size equal to the number of slots. The value in the array indicates the probability of selecting one of the slots for transmission. Initially, all the slots are assigned the same value, which means that no slot has higher probability than another.

v. Each sensor node is equipped with a battery, and the initial energy for all sensor nodes is the same.

\section{Network Hierarchy}


197 We build a GMAC protocol based on a network hierarchy depicted in Figure 2. The information

198

199

200

201

202

203

204

205

206

207

208

209

210

211

212

213

214

215

216

217

218

219

220

221

222

223

224

225

226

227

228

229

230

231

232

on environment, sensors, locations, and sinks are provided to the application responsible for managing the network. The information is analyzed, and the optimal cluster decomposition and cluster head assignment are generated and provided to the sinks by the SDN controller through the flow table. Each sink knows its clusters, and their cluster heads become responsible for collecting the data to send to the cloud. GMAC protocol operates within this sub-network that is assumed to be non-overlapping in the coverage zone when using low transmission mode, which is only enabled within cluster communication. In the beginning, the sink broadcasts an information frame that carries the cycle specification, slots, and their definitions. The details of this protocol are presented in the following sub-section.

\section{Energy Model}

Energy is consumed at each sensor whenever a data packet is sent or received. The consumed energy is calculated according to number of bits in the packet for transmission and receiving and the number of bits in the packet and the distance between the sender and receiver in the transmission case. We also assume that the sensors can operate in one of two modes. The first is the high energy mode for communicating between cluster heads and sinks, and the second is the low energy mode for communicating within clusters. This model is based on the radio energy dissipation model presented in Equations (1) and Equation (2) that are given in (Wang et al., 2017).

$$
\begin{gathered}
E_{T x}(k, d)=E_{T x-\text { elec }}(k)+T_{T x-\text { amp }}(k, d)= \\
\left\{\begin{array}{l}
E_{\text {elec }} * k+\varepsilon_{f s} * k * d^{2}, d \leq d_{0} \text { for low energy mode } \\
E_{\text {elec }} * k+\varepsilon_{m p} * k * d^{4}, d>d_{0} \text { for high energy mode }
\end{array}\right.
\end{gathered}
$$

To receive k bits' message, the energy consumption is given in Equation (2).

$$
E_{R X}(k)=E_{R X-\text { elec }}(k)=E_{\text {elec }} * k
$$

\section{Energy-aware back-off time}

One of the developed models of the protocol is the energy-aware back-off time which enables the node to consider its residuals energy. The approach uses the current energy in the node $E$, the minimum allowed energy $E_{\min }$, the maximum energy $E_{\max }$, the maximum and minimum values of $C W$ or $C W_{\max } C W_{\min }$ in a linear proportional model as it to select the best $C W E$ as it is given in Equation (3) which will be used in the back-off time calculation in Equation (4).

$$
C W E=\left(\frac{C W_{\max }-C W_{\min }}{E_{\max }-E_{\min }}\right)\left(E-E_{\min }\right)+C W_{\text {min }}
$$

Back off_Time $=$ Random $(0, C W E) \times$ SlotTime 
233

234

235

236

237

238

239

240

241

242

243

244

245

246

247

248

249

250

251

252

253

254

255

256

257

258

259

260

261

262

263

264

265

266

267

268

269

270

3

Considering that at certain point of time, the nodes will have different values of residual energies, then it is more likely when two nodes contribute in a collision when attempting to access the channel, they have different energies. Hence, the Packet Delivery Ratio (PDR) will get more chance to increase, and node will be more immune from wasting its energy in frequent collision. The suffix EA will be used to indicate to the protocol that uses this developed model.

\section{Protocol Design}

GMAC is a MAC scheduling protocol for WSN that is partitioned into multi-clusters and six cycles. Each cycle contains one frame, except cycle 1 that includes $N_{C}$ frames. Hence, the total number of frames is $5+N_{C}$. Next, we present the details of the cycles, the general protocol activity diagram and their corresponding frames.

1) Cycle 0. One-time cycle that occurs only at the beginning of the network. In this cycle, the frame is sent from the sink as a broadcast frame to identify the cluster heads, nodes, and the assignments. The frame is a broadcast frame, and the nodes are in low power mode. In case of packet loss within this cycle, the same settings of the previous cycles are used.

2) Cycle 1. A periodic cycle to send data from the nodes to the cluster head. The nodes operate in low coverage mode, and the frames in the cycle differ from one cluster to another according to cluster size and the group information. The frame of any cluster $i$ contains the number of slots according to Equation (5) and Equation (6). Each subframe $i$ has the size of
$N f_{i}=m \sum_{j=1}^{L} L_{j} N_{j}^{i}$
$L_{j}=\operatorname{maxGroup}-\mathrm{j}+1$

Where, maxGroup denotes the maximum number of groups in the sensors, and $\mathrm{j}$ denotes an index of the group. Low index is equivalent to high priority level given to the group.

$N_{j}^{i}$ denotes the number of nodes in cluster $i$ that have the priority level $L_{j}$. The number of slots that are assigned to priority level $L_{j}$ in sub-frame equals to $L_{j} N_{j}^{i}$ and denoted by $x g_{j}^{i}$. Thus,

$$
N f_{i}=\sum_{j=1}^{L} x g_{j}^{i} \times m
$$

The nodes inside group $j$ has the right to compete for its slot only inside the sub-frame $i$, avoiding wasted energy of the nodes competing with those of different groups.

Example 1: Assume a cluster i with combined four groups and five nodes in each group. The frame structure is 
271

272

273

274

275

276

277

278

279

280

281

282

283

284

285

286

287

288

289

290

291

292

293

294

295

296

297

298

299

300

301

302

303

304

305

306

307

308

$$
\begin{aligned}
& N f_{j}=5(1 \times m+2 \times m+3 \times m+4 \times m) \\
& \quad=5(1+2+3+4)=50 \quad, \text { where } \mathrm{m}=1 .
\end{aligned}
$$

The equation of $N_{f}$ can be generalized to consider the number of nodes $N_{i}$ in group i when the number of nodes differ in each group, such as

$$
N f_{j}=\sum_{j=1}^{L} L_{j} m \times N_{j}^{i}
$$

3) Cycle 2. A periodic cycle to send data from cluster heads to sinks. In this cycle, the cluster heads operate in high coverage mode while other nodes are in sleep mode. As provided in Equation (9), the frame type of this cycle is long and setup by the coordinator based on the cluster sizes and number. On the one hand, each cluster head has a predefined number of slots according to its cluster size. In this frame, cluster heads do not compete. On the other hand, the nodes in each cluster are in sleep mode while their cluster head is communicating with the sink, to prevent interference. The frame is decomposed of $N_{c}$ subframes where each is assigned to one cluster head. After the cluster head selection by the controller, each cluster head knows its dedicated subframes for transmission. The sub-frame sizes are determined on the basis of the number of nodes in their corresponding cluster and their groups. The frame consists of the number of slots equal to $N_{t f}$.

$$
N_{t f}=m \sum_{j=1}^{N c} \times \sum_{i=1}^{L j} L_{j} \times N_{i}=\sum_{j=1}^{N c} f_{j}
$$

Example 2: Assume a network of four clusters. Table 1 shows that each cluster contains different numbers of groups and nodes inside each group. Apply Equation (7) to calculate the total size of the sub-frame for the corresponding cluster when $m=1$. To explain the sequence or the network cycle in this example, we show the detail frame and sub-frames in Figure 3.

4) Cycle 3. A periodic cycle triggered every $T_{u}$ to update the energy status and packet delivery status of the nodes to their cluster heads. The cycle repeats after the NC number of cycles that are predefined by the application. Nodes work in low coverage mode. Only one frame, update frame 1 , is contained, and its length is the same as in cycle 1 .

5) Cycle 4. A periodic cycle triggered every $T_{u}$ to update the energy and PDR status of the nodes to the sink. In this cycle, a long frame is sent by cluster heads to the sink that has predefined slots. The cluster heads operate in high coverage mode while other nodes are in sleep mode. The frame type, update frame 2, is a long frame as provided in Equation (9).

6) Cycle 5. A periodic cycle triggered every $T_{u}$ and broadcasted by the sink to change the cluster 
heads based on the node energy consumption. Only one frame is contained, update frame 3 , to identify the new head of a certain cluster. In addition, the cycle contains an indicator variable that defines one of three possible states: State 1 allows contention only for nodes that have not occupied their slot, State 2 allows contention for all nodes, and State 3 does not allow any contention.

7) Network Cycle. Figure 3 shows that the network consists of six cycles. In cycles 0,1 , 4, and 5 , the nodes work in low power mode wherein the coverage radius is regarded as RA $\mathrm{m}$. This value assures no interference between nodes in one cluster with those in another cluster. In cycles 2 and 3, the cluster head works in high power mode in which the radius becomes RB and is responsible for sending data from the cluster head to the sink. Nodes in high power mode go on sleep mode except for the cluster head.

8) Protocol Activity Diagram. Figure 4 shows the activity diagram that provides sequential descriptions of the various cycles and tasks in the GMAC protocol. The class diagram starts with having a broadcast frame to inform all the nodes about clustering and the cluster head decision. This application layer makes this decision in the cloud based on various considerations, which is beyond the scope of this study. This broadcast frame includes information of each cluster of each node, its cluster head, and its dedicated slot in the TDMA cycles. The sizes of those cycles and contention access periods and their sizes are also defined.

Next, each cluster's nodes repeatedly send data to its cluster head using CAP period of ISA or IEEE frame. Subsequently, TDMA cycle is performed to send the data gathered by the cluster head to the sink in sequential manner. We define a time period $T_{u}$ that updates the cluster head and the sink on node energy status. This awareness enables maximum utilization of slots by updating the indicator variable that allows the nodes to increase this contention, and consequently, maximize its rewards. Hence, three consecutive cycles are triggered every $T_{u}$ : cycle 3 for having nodes updating its cluster head using TDMA about its energy and nodes occupation status, cycle 4 for having the cluster head update the sink about their cluster states using TDMA, and cycle 5 when the sink updates the entire network about the clustering decision and the indicator variable. The pseudocode of the whole protocol is presented in Algorithm 1.

Algorithm 1 Pseudo code of the GMAC-ISA/IEEE protocol

Input

Locations of sensors and their initial battery levels

Fixed sink

Start

while (sink is active)

1-the sink node sends broadcast frame to inform about cluster heads and cluster decomposition 
2-the node in each cluster sends data to its cluster heads using ISA/IEEE frame with CAP 3 -each cluster head sends its cluster data to the sink in its dedicated slots inside ISA/IEEE frame TDMA

4- if (time to update cluster head)

4.1 in each cluster, nodes will update the cluster head about their status using TDMA

4.2 cluster head updates sink about the cluster status using TDMA

4.3 sink updates clustering decision and indicator variables

else

go to 2

5-end

End

\section{EXPERIMENTAL DESIGN AND RESULTS}

346

347

348

349

350

351

352

353

354

355

356

357

358

359

360

361

362

363

364

365

366

367

368

369

370

This section provides the evaluation scenarios and experimental works for comparing our GMAC with the benchmarks IEEE 802.15.4, ISA 100.11a, and UDG (Yasari et al., 2017). GMAC has two variants, namely, GMAC-IEEE and GMAC-ISA. Hence, we have five protocols: GMAC-IEEE, GMAC-ISA, and UDG based on ISA 100.11a, IEEE 802.15.4, and ISA 100.11a.

\section{1) Experiment Design}

The simulation work uses MATLAB 2019b for evaluation. The experiment design is based on changing the inter-arrival time that indicates the offered load in the network. The range of the inter-arrival time changes from 0.1 to $5 \mathrm{sec}$. In each experiment, a set of scenarios are generated on the basis of changing two variables, the number of nodes in the network and the number of clusters. The number of nodes is $K=10,20, \ldots 100$, and the number of clusters is taken as $N_{c}=$ $1,2, . .10$. In addition, different priority groups are generated $L_{j}=1,2, . .10$. Moreover, the average number inside each cluster ranges from $\frac{K}{N_{c}}-\sigma$ to $\frac{K}{N_{c}}+\sigma$ where $\sigma$ denotes the diversity of the number of nodes in the clusters. Table 2 provides the simulation parameter. For channel fading, we use Nakagami, which is a method commonly used in the simulation of physical fading radio channels. Using the parameter, this distribution can model signal fading conditions ranging from extreme to mild, light to no fading, Beaulieu \& Cheng, 2005). The scenario details in terms of number of nodes and number of clusters are shown in Table 3 with the visualization of two scenarios in Figure 5(a) and Figure 5(b).

\section{2) Evaluation Results and Configuration}

The experimental results for the evaluation scenarios of Table 3 are provided in Figure 6 to Figure 17. First, we present the PDR of IEEE, GMAC-IEEE and GMAC-IEEE EA in Figure 6, and of ISA, GMAC-ISA and GMAC-ISA EA in Figure 7. GMAC-IEEE and GMAC-IEEE EA generate higher values of PDR compared with IEEE with slight superiority of GMAC-IEEE EA. This is interpreted by the geographical distribution awareness of G-MAC based protocols which 
371 enable less collision due to dividing them into geographical clusters with less overlap of their 372 coverage compared with one cluster contention in IEEE.

373

374 - Packet Delivery Ratio. Both GMAC protocols generate a PDR between $90 \%$ and $100 \%$,

375 whereas the PDR of IEEE is as low as $75 \%$ in scenarios $3-150$ and as low as $30 \%$ in

376

377 scenarios 4-500. Similarly, higher values of PDR for GMAC-ISA and GMAC-ISA EA are

378 obtained compared with basic ISA. ISA and UDG have lower PDR for scenarios 3-400 and

379 $5-400$. This is interpreted by the high number of nodes that are distributed in physical clusters

380 where ISA and UDG do not consider clustering them in the MAC scheduling. This causes

381

382 pressure on the sink. In GMAC with clusters and cluster head, the pressure is mitigated. Moreover, higher PDR is generated from IEEE-based protocols in Figure 6 compared with

383 the PDR generated from ISA-based protocol in Figure 7. This is interpreted by the packet

384 385 lifetime of the latter, which causes less opportunity of sending packets compared with IEEE protocol that does not add lifetime on the packets.

- End-to-end Delay. The second metric that is generated is end-to-end (e2e) delay, which is shown in Figure 8 for IEEE-based protocols and in Figure 9 for ISA-based protocol. In all scenarios in Figure 8, there is a slight difference between GMAC-IEEE's e2e delay and IEEE, indicating the competitive performance between the approaches in terms of the delay. In Figure 9, the difference in the delay between ISA GMAC-based protocols and ISA is higher that the corresponding results of IEEE, which is interpreted that the packet lifetime shows more influence in ISA experiment in reducing the delay compared with IEEE. In some scenarios, GMAC has lower e2e delay than the original IEEE. For example, the least e2e delay for GMAC-IEEE and GMAC-IEEE EA is for scenario 5-150, that is, 10s, which is lower than $3 \mathrm{~s}$ of the least e2e delay of IEEE. This is interpreted by the capability of GMAC 397 in enabling higher successful transmission with less number of trials compared with IEEE.

- Energy Consumption. The energy consumption is depicted in Figure 10 and Figure 11 for GMAC-based approaches and the original IEEE and ISA. In Figure 10, the energy consumption is much lower for GMAC-IEEE EA, and GMAC-IEEE than IEEE. We also observe that IEEE consumes the highest energy for all scenarios of 5 clusters, namely, 5-200, $5-300$, and 5-400 with some differences caused by the random changing in the geographical distribution of the node. However, GMAC-based protocols are less affected by the change in the number of nodes and number of clusters, and their energy consumption level is below 100 $\mathrm{W} / \mathrm{h}$ for all scenarios. Furthermore, we observe in scenario 5-300 that the energy consumption is $28.1 \mathrm{~W} / \mathrm{h}$ for GMAC-IEEE EA and GMAC-IEEE but around $578 \mathrm{~W} / \mathrm{h}$ for IEEE. Similarly, in Figure 11, the energy consumption for ISA-based protocols, that is, ISA and UDG, are the most consuming for scenarios 5-150, 5-200, and 5-300, indicating the effect of diverse localization area of the nodes and lack of grouping them within clusters on the energy consumption. However, GMAC-ISA is less affected because of its clustering-aware 
411

412

413

414

415

416

417

418

419

420

421

422

423

424

425

426

427

428

429

430

431

432

433

434

435

436

437

438

439

440

441

442

443

444

445

446

447

448

449

450

scheduling. Another observation is that GMAC-ISA EA has high energy consumption in some scenarios, such as 3-400, which is interpreted by its behavior in exploiting the residual energy of the nodes for sending that may cause more collision when the nodes reach a state of low energy level in the entire network. Another interpretation factor is the differences in the geographical positions of the nodes that cause non-linear energy consumption profile. More specifically, the changing in the node locations is another factor that causes variation in the energy consumption and to the number of nodes. Furthermore, the latter factor has non-linear component in the overall energy consumption due to the piecewise nature of the energy consumption formula.

- Network Lifetime. The other metric that is generated is the lifetime, which indicates the experiment time until the first node exits the network. In Figure 12, GMAC-IEEE and GMAC-IEEE EA experience the same lifetime, which is in general longer than the lifetime of IEEE. This is similar to the behavior of GMAC-ISA and GMAC-ISA EA compared with ISA as in Figure 13. UDG has a longer lifetime. The pro-organizing aspect of GMAC interpreted the behavior before enabling contention compared with the other protocol that limits the number of collision and prolong the lifetime of the network. The number of retransmissions, which indicates the collision level in the network, is depicted in Figure 14 and Figure 15. The retransmission numbers for IEEE and ISA are higher than those of GMAC and GMAC EA (IEEE and ISA). This is correlated with the energy consumption of the protocols. As stated earlier, this indicates the role of clustering-aware GMAC in mitigating the collisions in the contention.

- Throughput. The last metric that is generated is the throughput, which indicates how much of the bandwidth is exploited in producing successful transmission. The results of this metric for IEEE-based and ISA-based protocols are depicted in Figure 16 and Figure 17, respectively. Both figures reveal that GMAC and GMAC EA have higher throughput than the basic protocols including UDG, regardless of the scenario. The highest achieved throughput by GMAC-IEEE EA and GMAC-IEEE is 539kbps and 529kbps, respectively, compared with only $68.4 \mathrm{kbps}$ by IEEE. Similarly, Figure 17 demonstrates that GMAC-ISA and GMAC-ISA EA have accomplished throughput of $352.7 \mathrm{kbps}$ and $350.2 \mathrm{kbps}$, compared with $19.8 \mathrm{kbps}$ and $13.8 \mathrm{kbps}$ by ISA and UDG, respectively. Obviously, the high throughput is achieved because of the effective management architecture of nodes access to the medium and its tight relation with the nodes geographical location which is an ignored factor in the original IEEE and ISA.

\section{Conclusions}

This study proposes a novel protocol for MAC layer in IoT network considering three aspects of operations: low energy level of nodes, high number of nodes, and need of grouping the nodes according to their priorities. This protocol is considered distinct from state-of-the art MAC protocols for IoT, such as IEEE 802.15.4 and ISA 100.11a. In addition, GMAC is suitable to 
451

452

453

454

455

456

457

458

459

460

461

462

463

464

465

466

467

468

469

470

471

472

473

474

475

476

477

478

479

480

481

482

483

484

485

486

487

488

489

490

491

work using SDN technology where a control plane is separated from the data plane and the network is managed by an SDN controller. This is because the protocol requires adaptability to the geographic node distribution by incorporating clustering awareness in the frames and cycles. In addition, GMAC decomposes the network into cycles to enable efficient medium sharing and competition by activating channel access attempts within the cluster that is supposed to be isolated from other clusters in terms of communication collision when a low energy level is used. This is sufficient to transfer packets from nodes to cluster heads while transferring packets to the sink uses dedicated cycles by cluster heads. GMAC is compared with state-of-the-art protocols with respect to various scenarios in terms of number of nodes and priority levels. A clear superiority is observed in PDR, energy consumption, and competitive performance in terms of e2e delay. GMAC protocols generate a PDR higher than $90 \%$, whereas the PDR of benchmark is as low as $75 \%$ in some scenarios and $30 \%$ in others. In addition, GMAC protocols has lower e2e delay than the least e2e delay of IEEE with a difference of 3s. Regarding energy consumption, the consumed energy is $28.1 \mathrm{~W} / \mathrm{h}$ for GMAC-IEEE EA and GMAC-IEEE, which is lower than that of IEEE 802.15.4 $(578 \mathrm{~W} / \mathrm{h})$ in certain scenarios. The highest achieved throughput by GMAC-IEEE EA and GMAC-IEEE is $539 \mathrm{kbps}$ and $529 \mathrm{kbps}$, respectively, compared with only $68.4 \mathrm{kbps}$ by IEEE. As limitations for the work, we state that it requests that the nodes are distributed in a wide geographical area where their various clusters have no overlapping in the coverage, so the intra-cluster operations are executed without collisions with other clusters. Second, it assumes that the nodes are stationary, which does not change the clustering decision. However, it enables dynamic reallocation of the cluster head. Future work can develop more energy-saving techniques and incorporate machine learning for channel access. Another future work is to implement hardware for validating the new protocol and evaluate it in real-world scenarios. Developing the work to include channel hopping is an additional future work.

\section{References}

Al-Majhad, H. G., Bramantoro, A., Syamsuddin, I., Yunianta, A., Basori, A. H., Prabuwono, A. S., \& Barukab, O. M. (2018). A traffic congestion framework for smart riyadh city based on iot services. International Journal of Advanced Computer Science and Applications, 9(4), 292-303.

Beaulieu, N. C., \& Cheng, C. (2005). Efficient Nakagami-m Fading Channel Simulation. IEEE Transactions on Vehicular Technology, 54(2), 413-424. doi:10.1109/tvt.2004.841555

Benzerbadj, A., Kechar, B., Bounceur, A., \& Hammoudeh, M. (2018). Surveillance of sensitive fenced areas using duty-cycled wireless sensor networks with asymmetrical links. Journal of Network and Computer Applications, 112, 41-52. doi: 10.1016/j.jnca.2018.03.027

Farayev, B., Ucar, S., Sadi, Y., \& Coleri, S. (2020). Energy efficient robust scheduling of periodic sensor packets for discrete rate based wireless networked control systems. Ad Hoc Networks, 106. doi: 10.1016/j.adhoc.2020.102203

Florencio, H., Doria Neto, A., \& Martins, D. (2020). ISA 100.11a Networked Control System Based on Link Stability. Sensors (Basel), 20(18). doi:10.3390/s20185417 
492

493

494

495

496

497

498

499

500

501

502

503

504

505

506

507

508

509

510

511

512

513

514

515

516

517

518

519

520

521

522

523

524

525

526

527

528

529

530

531

532

533

534

535

536
Gamal, M., Sadek, N., Rizk, M. R. M., \& Ahmed, M. A. E. (2020). Optimization and modeling of modified unslotted CSMA/CA for wireless sensor networks. Alexandria Engineering Journal, 59(2), 681-691. doi: 10.1016/j.aej.2020.01.035

Hassan, A. A.-h., Shah, W. M., Habeb, A.-h. H., Othman, M. F. I., \& Al-Mhiqani, M. N. (2020). An improved energy-efficient clustering protocol to prolong the lifetime of the WSNbased IoT. IEEE Access, 1-1. doi:10.1109/access.2020.3035624

Hassan, R., Qamar, F., Hasan, M. K., Aman, A. H. M., \& Ahmed, A. S. (2020). Internet of Things and Its Applications: A Comprehensive Survey. Symmetry, 12(10). doi:10.3390/sym 12101674

Henna, S., \& Sarwar, M. A. (2018). An Adaptive Backoff Mechanism for IEEE 802.15.4 Beacon-Enabled Wireless Body Area Networks. Wireless Communications and Mobile Computing, 2018, 1-15. doi:10.1155/2018/9782605

Herrmann, M. J., \& Messier, G. G. (2018). Cross-Layer Lifetime Optimization for Practical Industrial Wireless Networks: A Petroleum Refinery Case Study. IEEE Transactions on Industrial Informatics, 14(8), 3559-3566. doi:10.1109/tii.2018.2819678

Keswani, B., Mohapatra, A. G., Mohanty, A., Khanna, A., Rodrigues, J. J. P. C., Gupta, D., \& de Albuquerque, V. H. C. (2018). Adapting weather conditions based IoT enabled smart irrigation technique in precision agriculture mechanisms. Neural Computing and Applications, 31(S1), 277-292. doi:10.1007/s00521-018-3737-1

Memon, I., Shaikh, R. A., Hasan, M. K., Hassan, R., Haq, A. U., Zainol, K. A., \& Shaukat, S. (2020). Protect Mobile Travelers Information in Sensitive Region Based on Fuzzy Logic in IoT Technology. Security and Communication Networks, 2020, 1-12. doi:10.1155/2020/8897098

Muthuramalingam, S., Bharathi, A., Rakesh kumar, S., Gayathri, N., Sathiyaraj, R., \& Balamurugan, B. (2019). IoT Based Intelligent Transportation System (IoT-ITS) for Global Perspective: A Case Study. In Internet of Things and Big Data Analytics for Smart Generation (pp. 279-300).

Nassar, M. A., Luxford, L., Cole, P., Oatley, G., \& Koutsakis, P. (2019). The Current and Future Role of Smart Street Furniture in Smart Cities. IEEE Communications Magazine, 57(6), 68-73. doi:10.1109/mcom.2019.1800979

Onasanya, A., \& Elshakankiri, M. (2019). Smart integrated IoT healthcare system for cancer care. Wireless Networks. doi:10.1007/s11276-018-01932-1

Osamy, W., El-Sawy, A. A., \& Khedr, A. M. (2019). Effective TDMA scheduling for tree-based data collection using genetic algorithm in wireless sensor networks. Peer-to-Peer Networking and Applications, 13(3), 796-815. doi:10.1007/s12083-019-00818-z

Patel, N. R., \& Kumar, S. (2017). Enhanced Clear Channel Assessment for Slotted CSMA/CA in IEEE 802.15.4. Wireless Personal Communications, 95(4), 4063-4081. doi:10.1007/s11277-017-4042-5

Raptis, T. P., Passarella, A., \& Conti, M. (2020). A Survey on Industrial Internet With ISA100 Wireless. IEEE Access, 8, 157177-157196. doi:10.1109/access.2020.3019665

Sadeq, A. S. H., Rosilah; Mahdi, Ahmed. (2018). Enhanced MQTT for Providing QoS in Internet of Things (IoT): A Study. Advanced Science Letters, 24, 5199-5203.

Satrya, G. B., \& Shin, S. Y. (2020). Evolutionary computing approach to optimize superframe scheduling on industrial wireless sensor networks. Journal of King Saud University Computer and Information Sciences. doi: 10.1016/j.jksuci.2020.01.014

Peer] Comput. Sci. reviewing PDF | (CS-2021:06:62075:2:0:NEW 2 Sep 2021) 
537

538

539

540

541

542

543

544

545

546

547

548

549

550

551

552

553

554

555

556

557

558

559

560

561

562

563

564

565

566

567

568

569

570

571

572

573

574

Shrestha, B., Hossain, E., \& Choi, K. W. (2014). Distributed and Centralized Hybrid CSMA/CATDMA Schemes for Single-Hop Wireless Networks. IEEE Transactions on Wireless Communications, 13(7), 4050-4065. doi:10.1109/twc.2014.2327102

Sotenga, P. Z., Djouani, K., \& Kurien, A. M. (2020). Media Access Control in Large-Scale Internet of Things: A Review. IEEE Access, 8, 55834-55859. doi:10.1109/access.2020.2982357

Srikrishnaswetha, K., Kumar, S., \& Rashid Mahmood, M. (2019). A Study on Smart Electronics Voting Machine Using Face Recognition and Aadhar Verification with IOT. In Innovations in Electronics and Communication Engineering (pp. 87-95).

Uchiteleva, E., Shami, A., \& Refaey, A. (2017). Virtualization of Wireless Sensor Networks Through MAC Layer Resource Scheduling. IEEE Sensors Journal, 17(5), 1562-1576. doi:10.1109/jsen.2016.2643670

Wang, J., Cao, Y., Li, B., Kim, H.-j., \& Lee, S. (2017). Particle swarm optimization-based clustering algorithm with mobile sink for WSNs. Future Generation Computer Systems, 76, 452-457. doi: 10.1016/j.future.2016.08.004

Wang, L., Liu, Y., \& Yin, Z. (2018). A Hybrid TDMA/CSMA-Based Wireless Sensor and Data Transmission Network for ORS Intra-Microsatellite Applications. Sensors (Basel), 18(5). doi:10.3390/s18051537

Wu, F., Wu, T., \& Yuce, M. R. (2018). An Internet-of-Things (IoT) Network System for Connected Safety and Health Monitoring Applications. Sensors (Basel), 19(1). doi:10.3390/s19010021

Yao, C., Wu, S., Liu, Z., \& Li, P. (2019). A deep learning model for predicting chemical composition of gallstones with big data in medical Internet of Things. Future Generation Computer Systems, 94, 140-147. doi: 10.1016/j.future.2018.11.011

Yaqoob, I., Hashem, I. A. T., Ahmed, A., Kazmi, S. M. A., \& Hong, C. S. (2019). Internet of things forensics: Recent advances, taxonomy, requirements, and open challenges. Future Generation Computer Systems, 92, 265-275. doi: 10.1016/j.future.2018.09.058

Yasari, A. K. I., Latiff, L. A., Dziyauddin, R. A., Lilo, M. A., Aljeroudi, Y., \& Atee, H. A. (2017). Flexible online multi-objective optimization framework for ISA100.11a standard in beacon-enabled CSMA/CA mode. Computers \& Electrical Engineering, 64, 537-551. doi: 10.1016/j.compeleceng.2017.02.024

Zhang, C. (2020). Design and application of fog computing and Internet of Things service platform for smart city. Future Generation Computer Systems, 112, 630-640. doi: 10.1016/j.future.2020.06.016

Zieliski, Z., Chudzikiewicz, J., \& Furtak, J. (2019). An Approach to Integrating Security and Fault Tolerance Mechanisms into the Military IoT. In Security and Fault Tolerance in Internet of Things (pp. 111-128).

Peer) Comput. Sci. reviewing PDF | (CS-2021:06:62075:2:0:NEW 2 Sep 2021) 
Figure 1

Application of IoT-WSN in health care using SDN technology. 


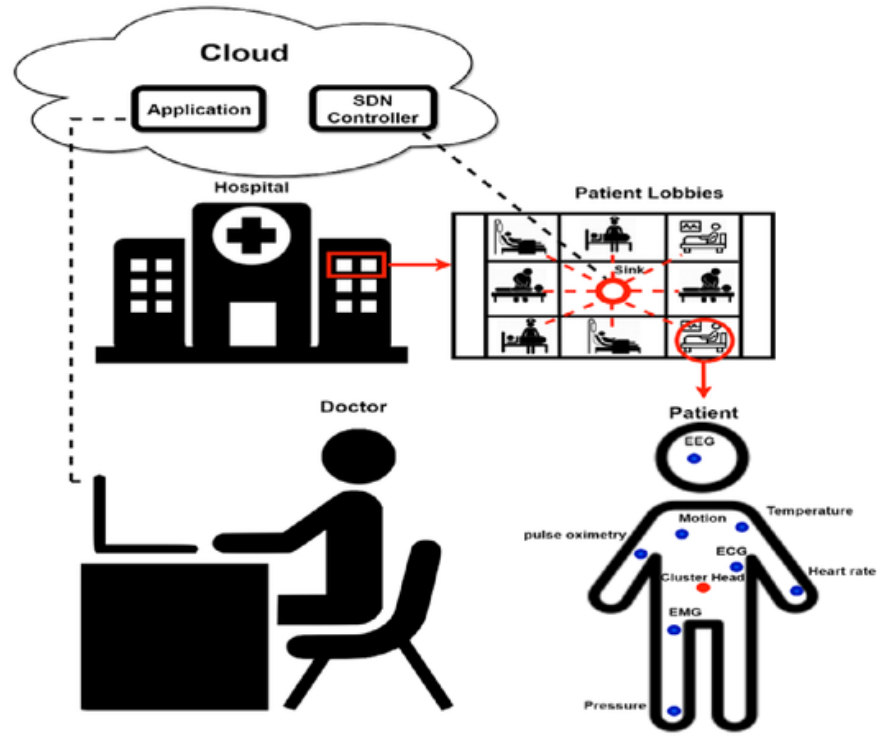


Figure 2

Conceptual diagram of the network hierarchy. 


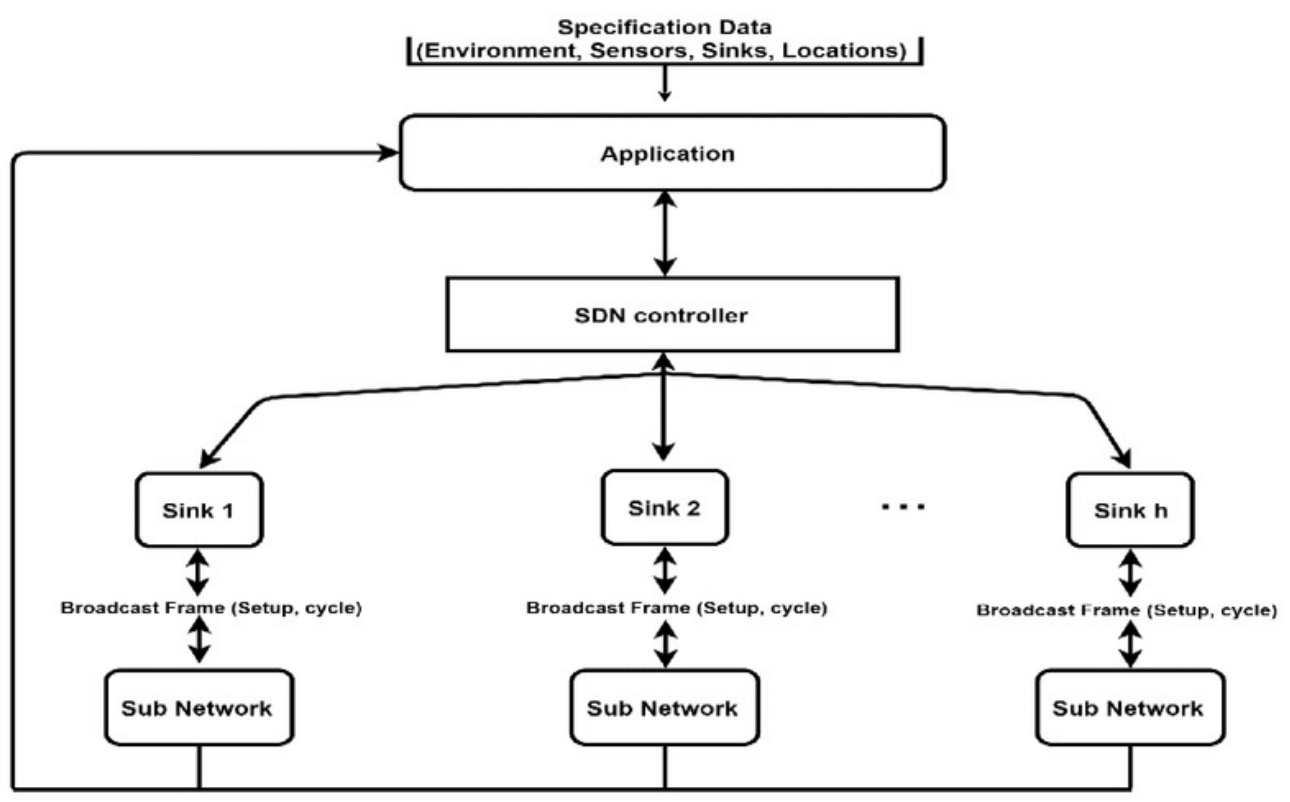


Figure 3

Total network cycles with the corresponding frame 


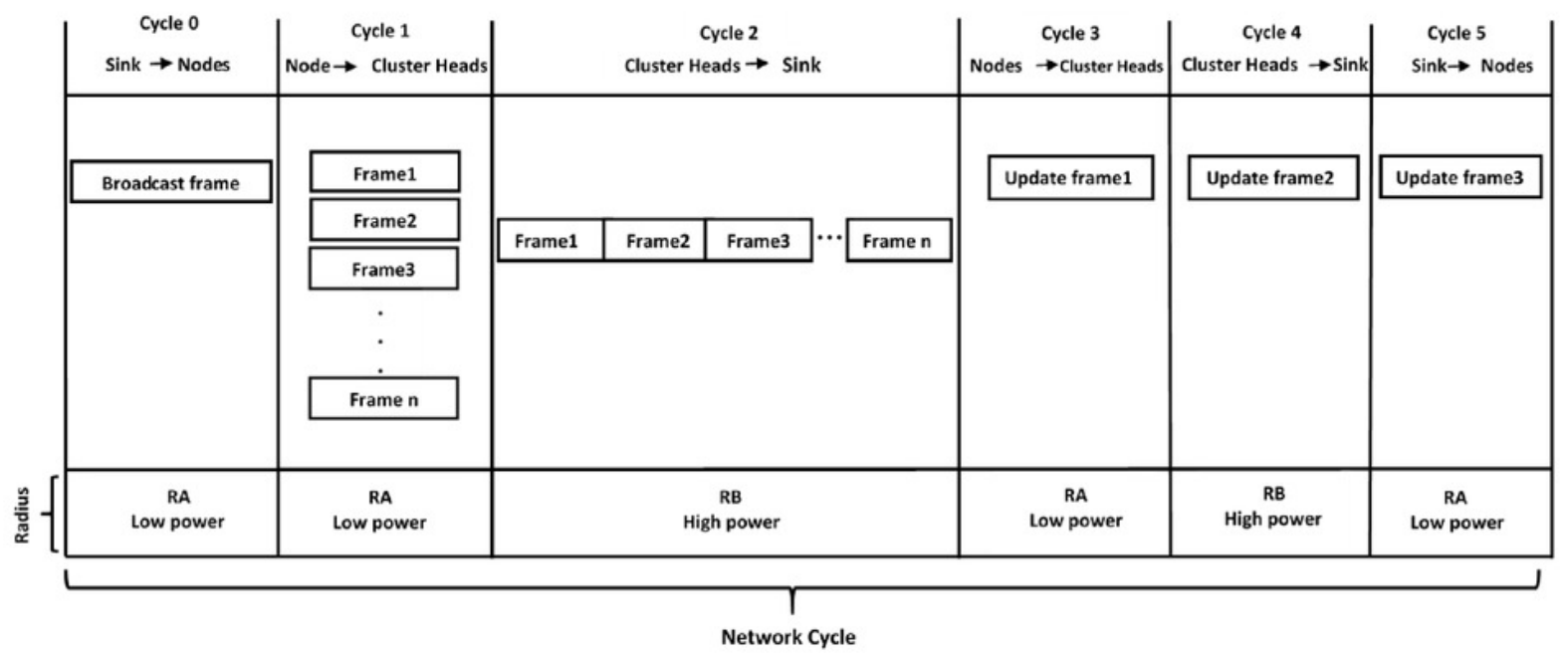


Figure 4

Activity diagram of GMAC protocol. 


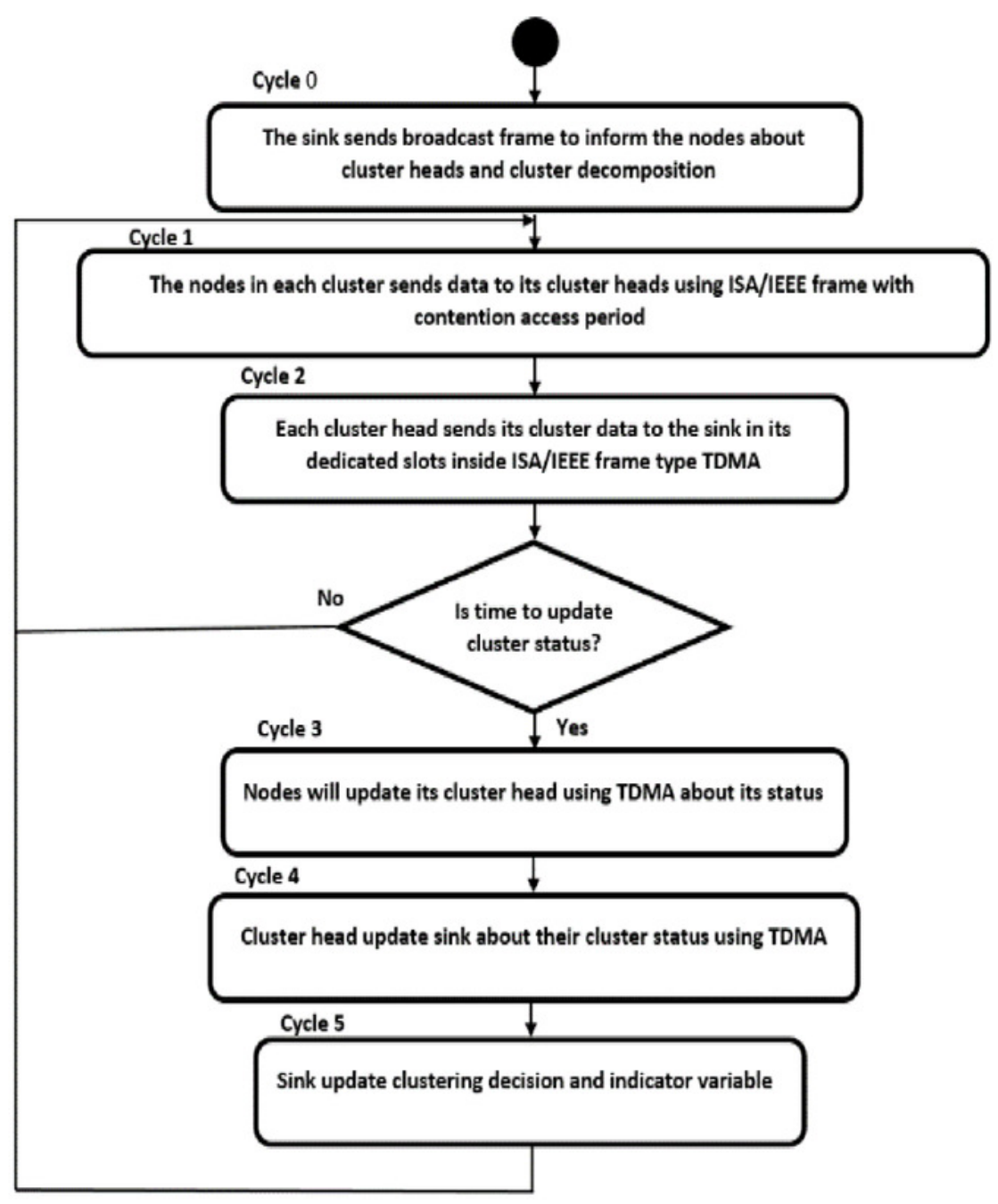




\section{Figure 5}

Visualization scenario with number of clusters: 3 and 5; and number of nodes: 300 


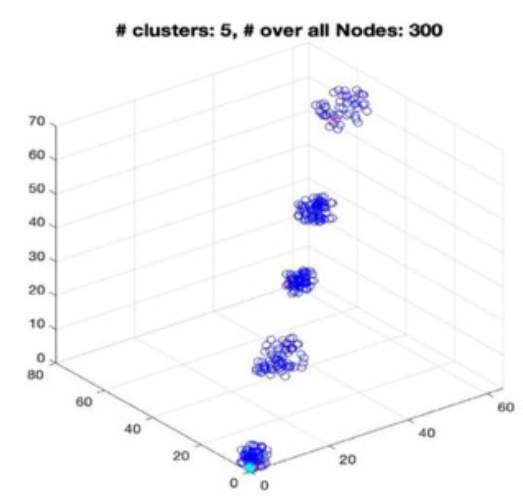

(a)

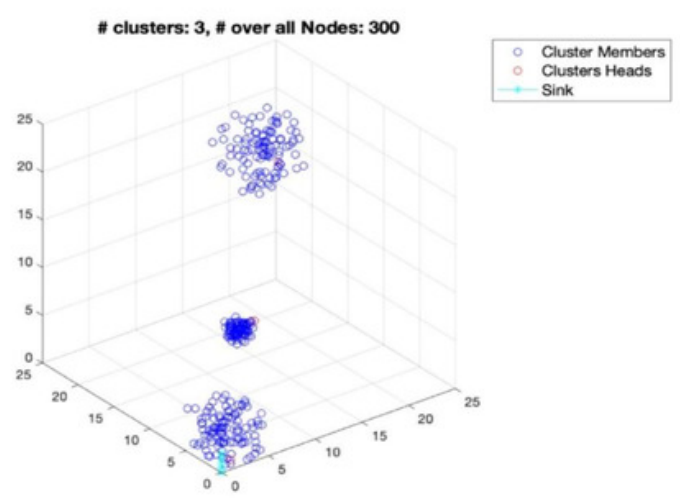

(b) 
Figure 6

PDR for GMAC and GMAC EA where the basic protocol is IEEE 


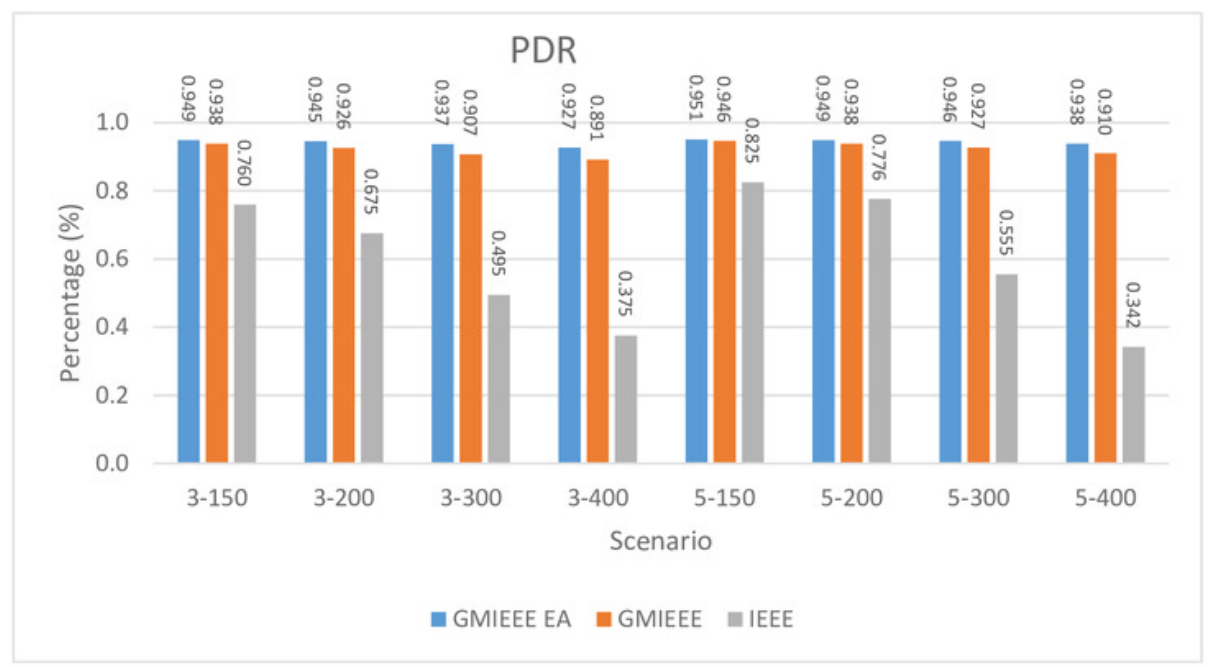


Figure 7

PDR for GMAC and GMAC EA where the basic protocol is ISA. 


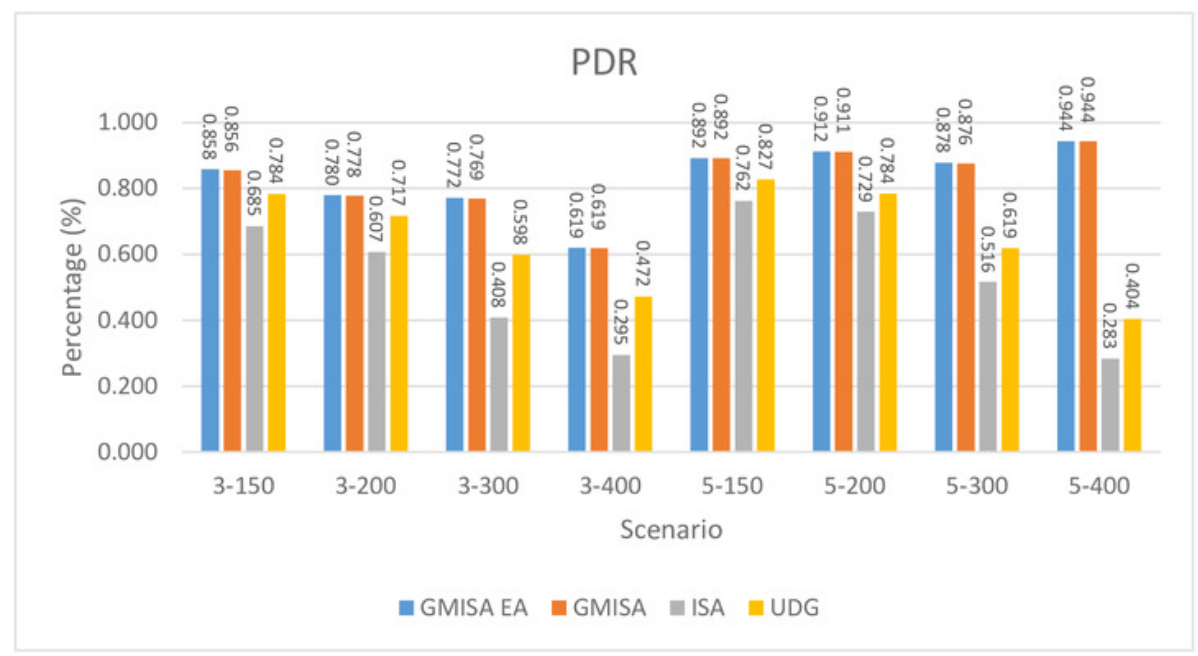


Figure 8

e2e delay for GMAC and GMAC EA where the basic protocol is IEEE 


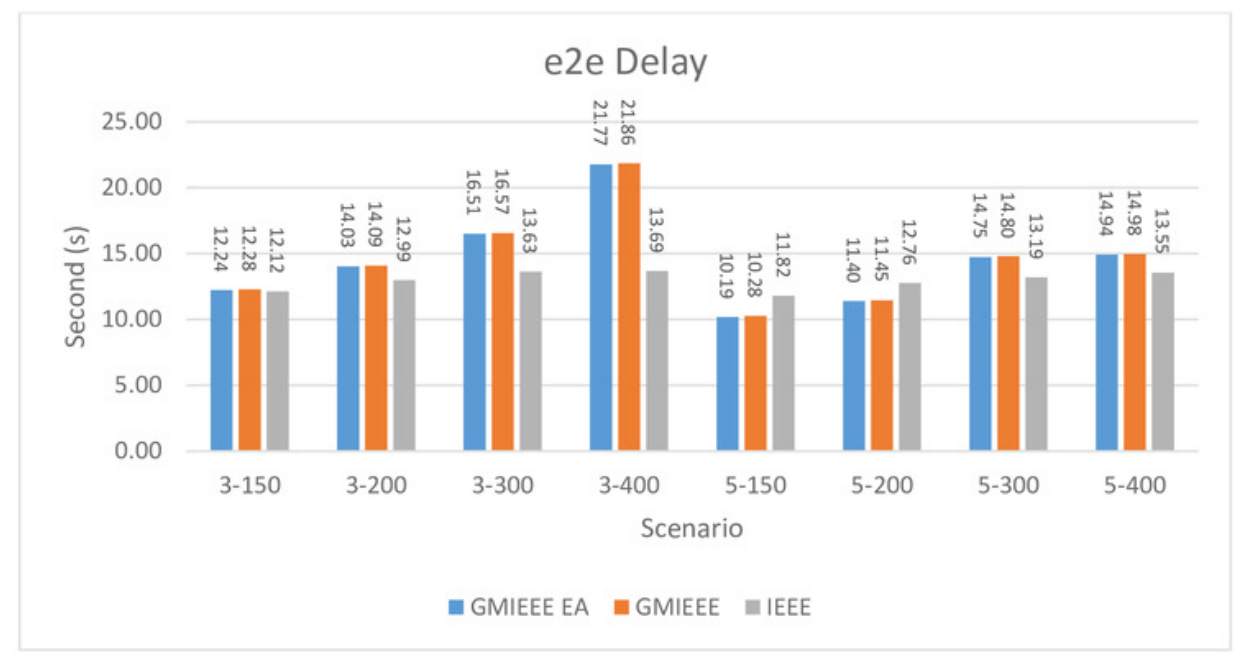


Figure 9

e2e delay for GMAC and GMAC EA -where the basic protocol is ISA. 


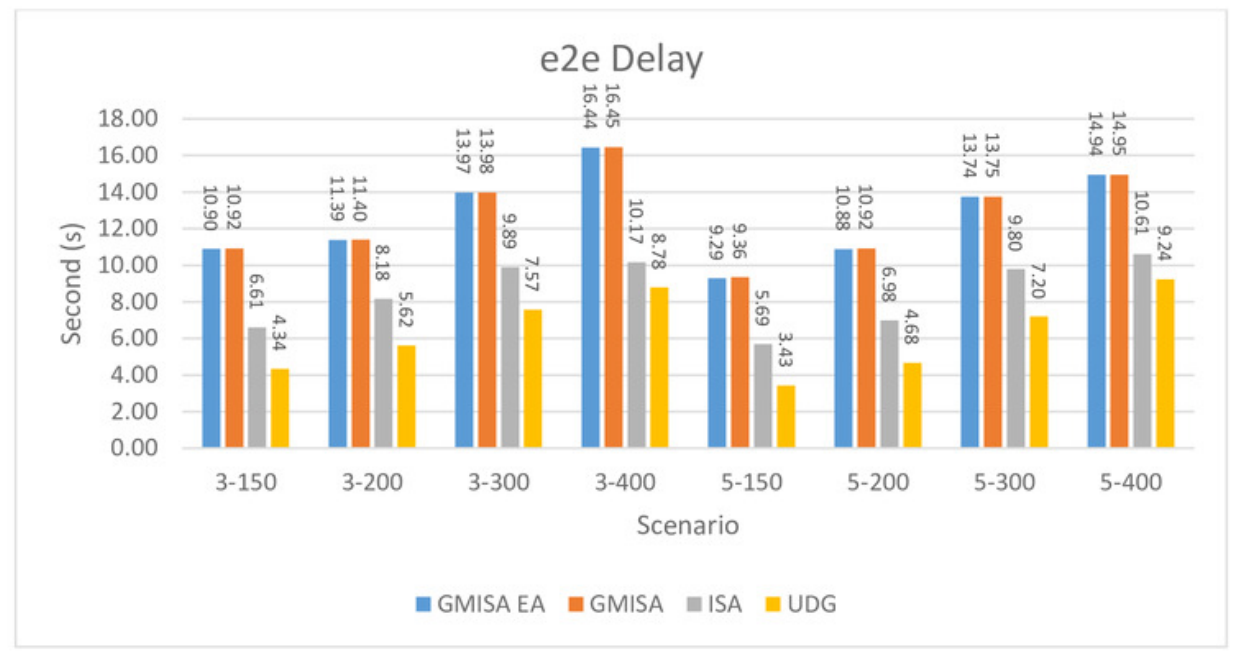


Figure 10

Energy consumption for GMAC and GMAC EA where the basic protocol is IEEE 


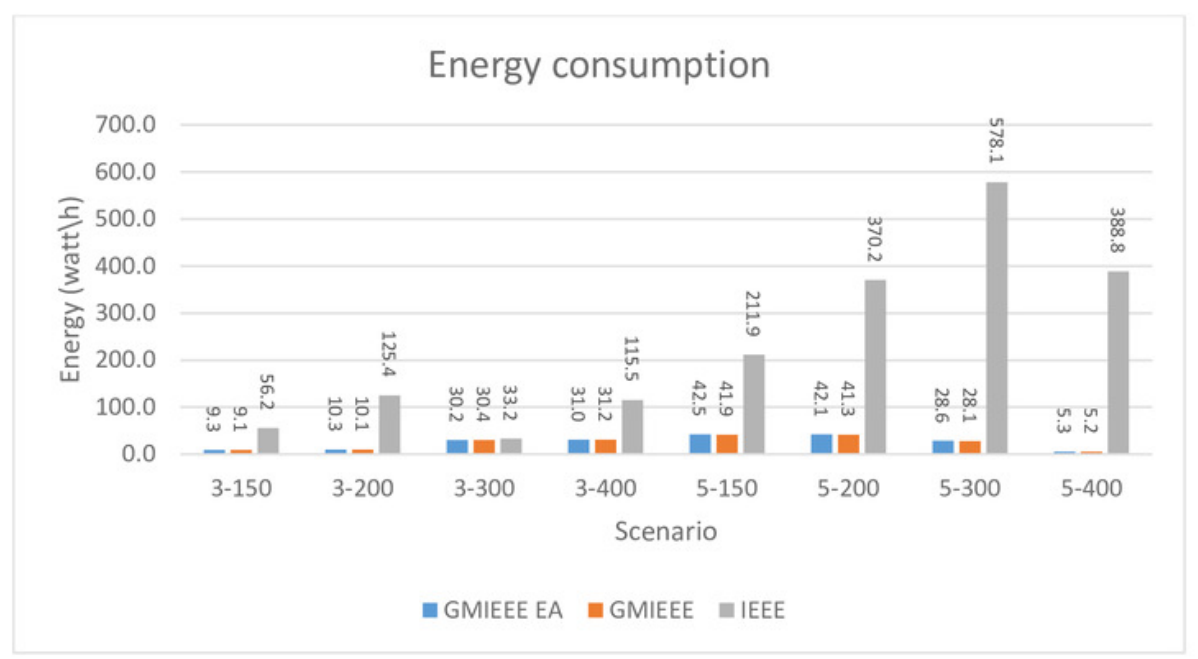


Figure 11

Energy consumption for GMAC and GMAC EA where the basic protocol is ISA. 


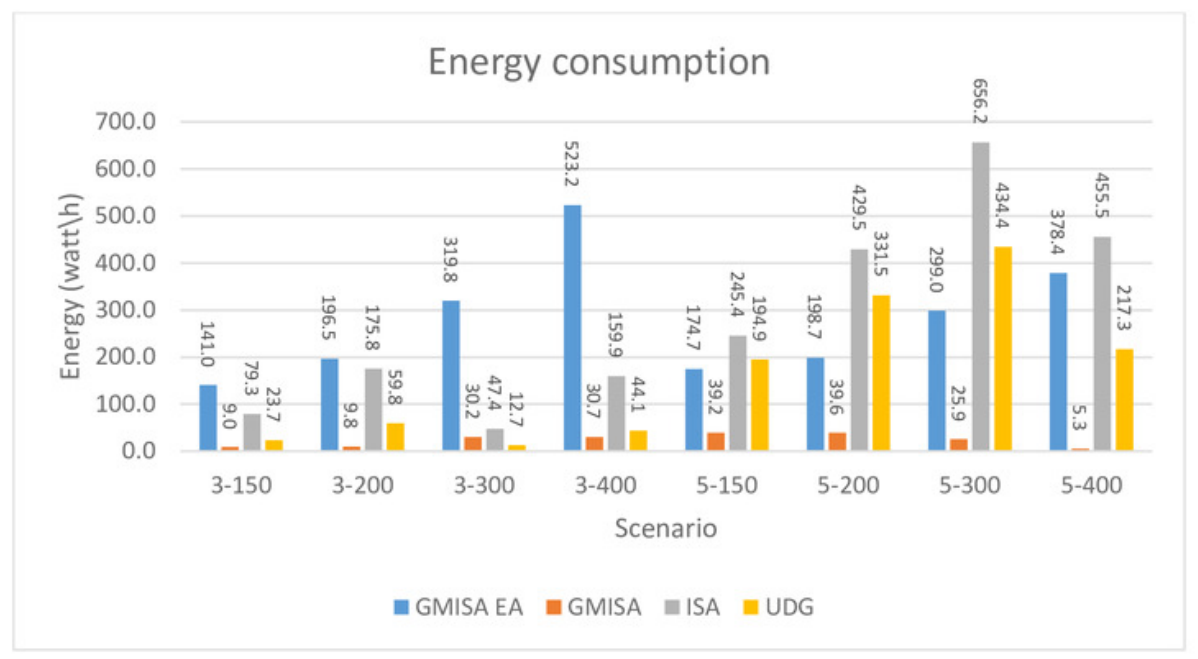




\section{Figure 12}

. Lifetime for GMAC and G-MAC-EA where the basic protocol is IEEE 


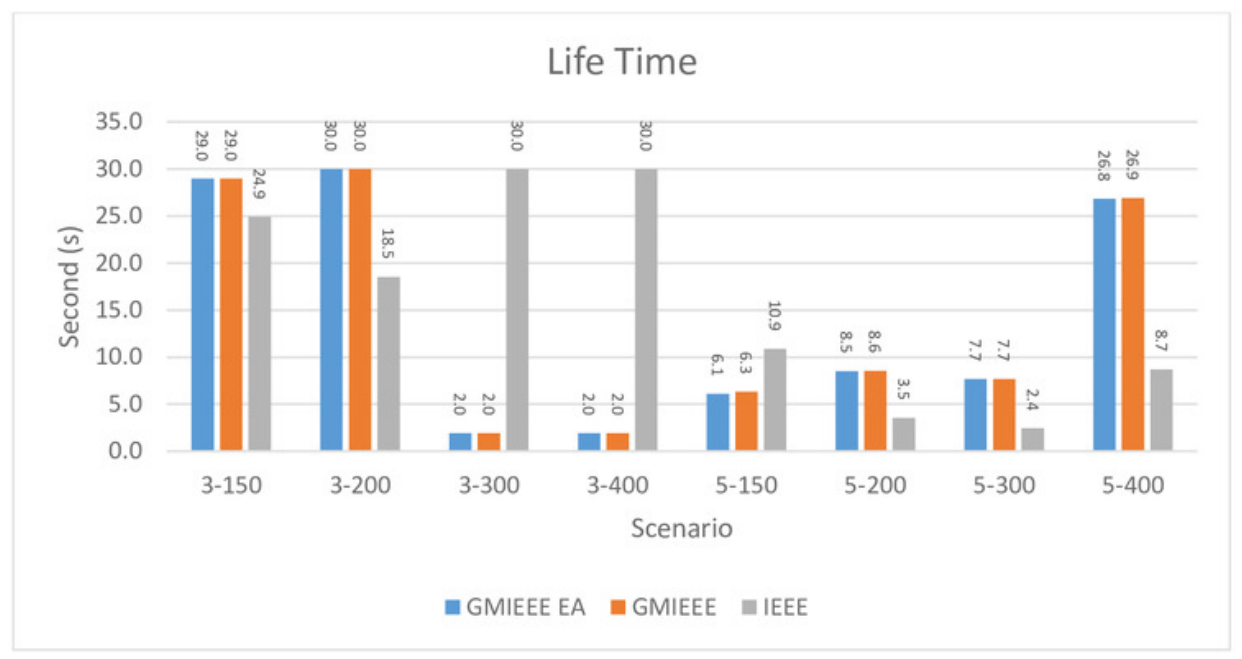


Figure 13

. Lifetime for GMAC and G-MAC-EA where the basic protocol is ISA 


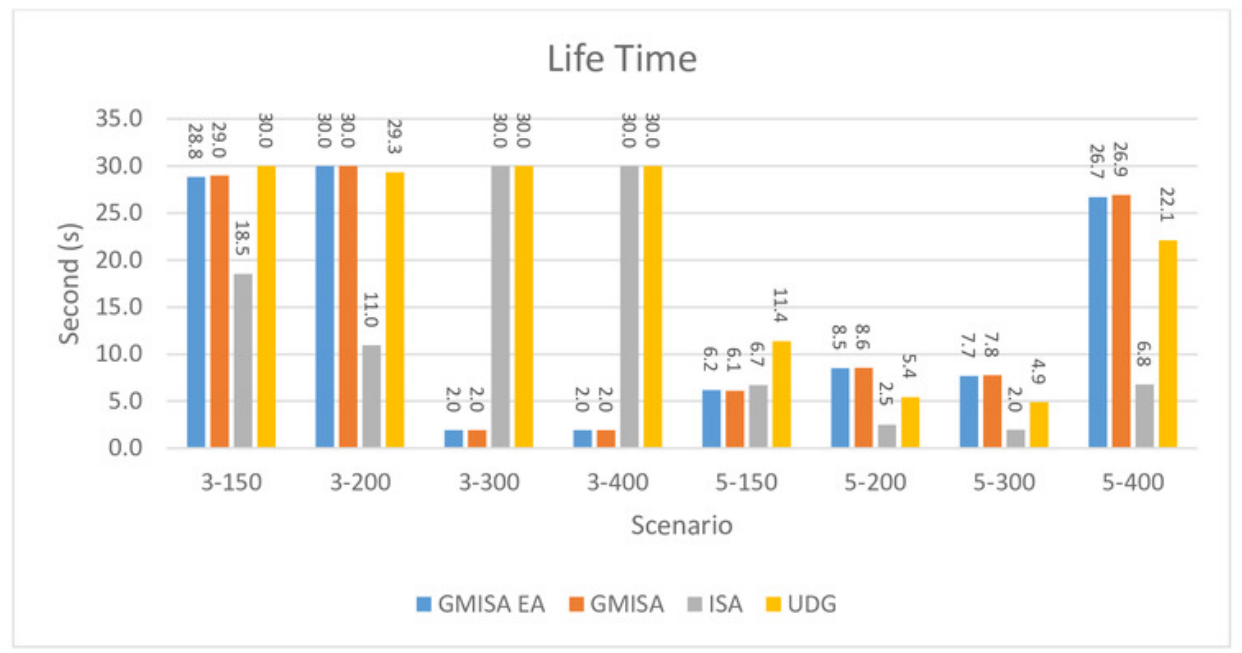




\section{Figure 14}

Retransmission times for GMAC and GMAC EA where the basic protocol is IEEE 


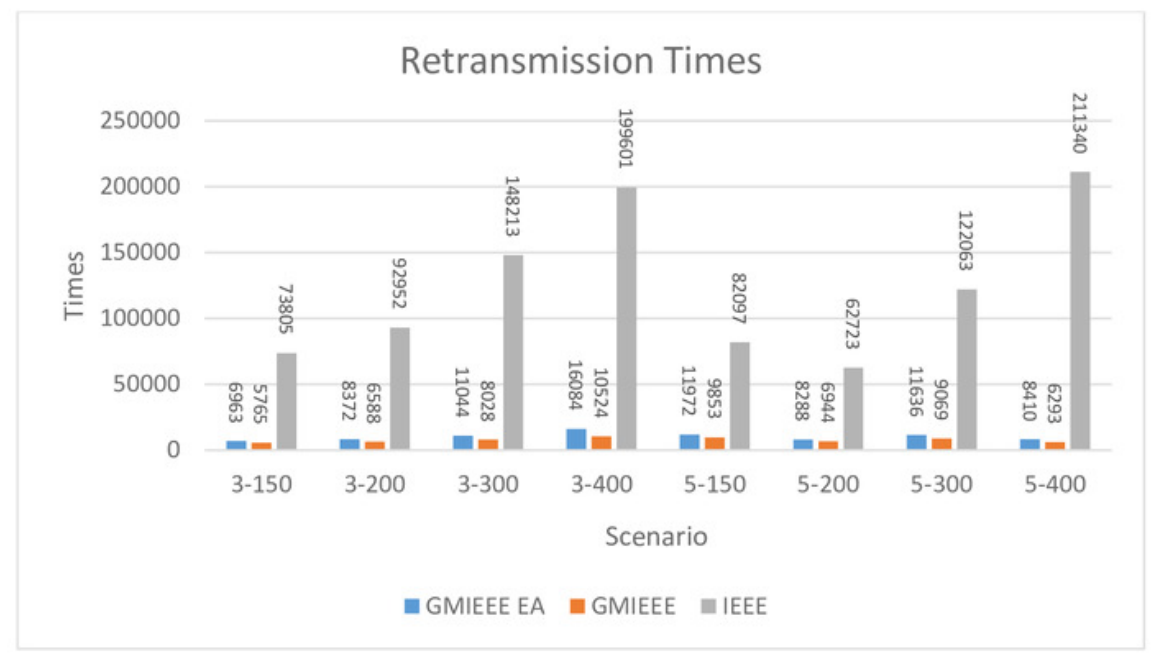




\section{Figure 15}

Retransmission times for GMAC and GMAC EA where the basic protocol is ISA. 


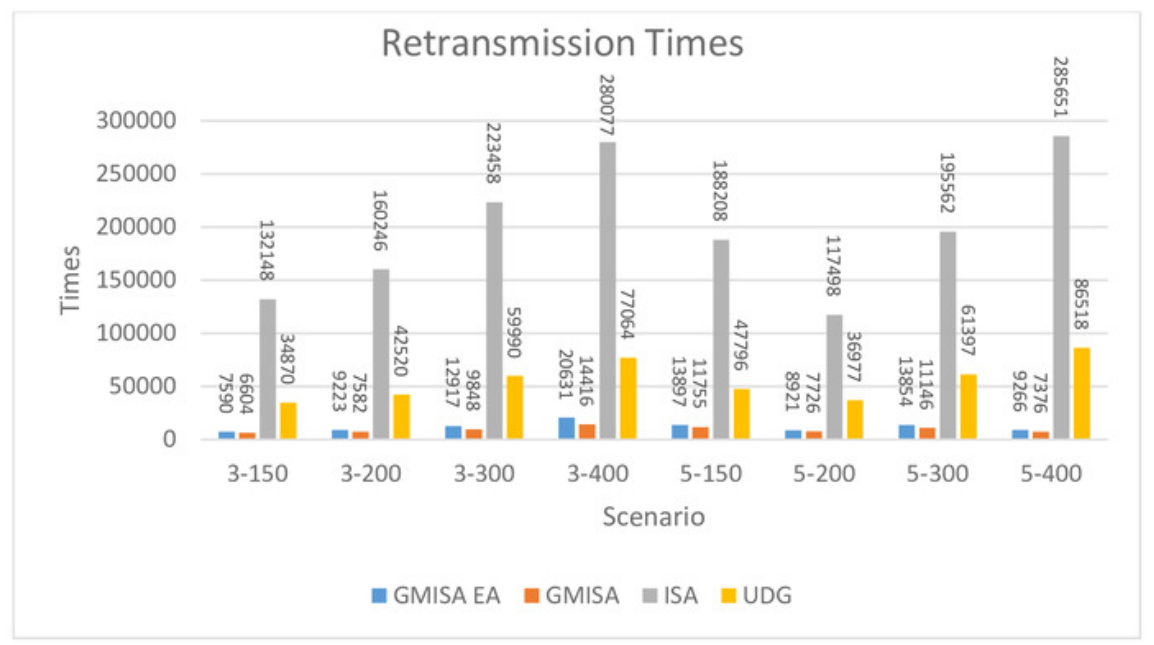




\section{Figure 16}

Throughput for GMAC and GMAC EA where the basic protocol is IEEE 


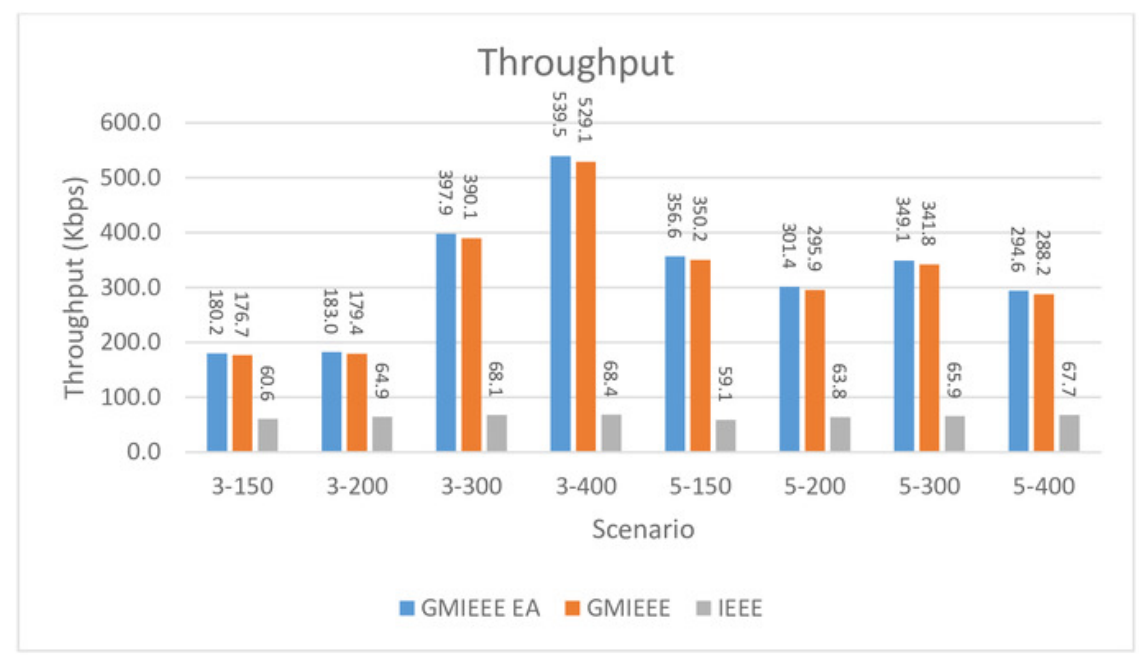


Figure 17

Throughput for GMAC and GMAC EA where the basic protocol is ISA. 


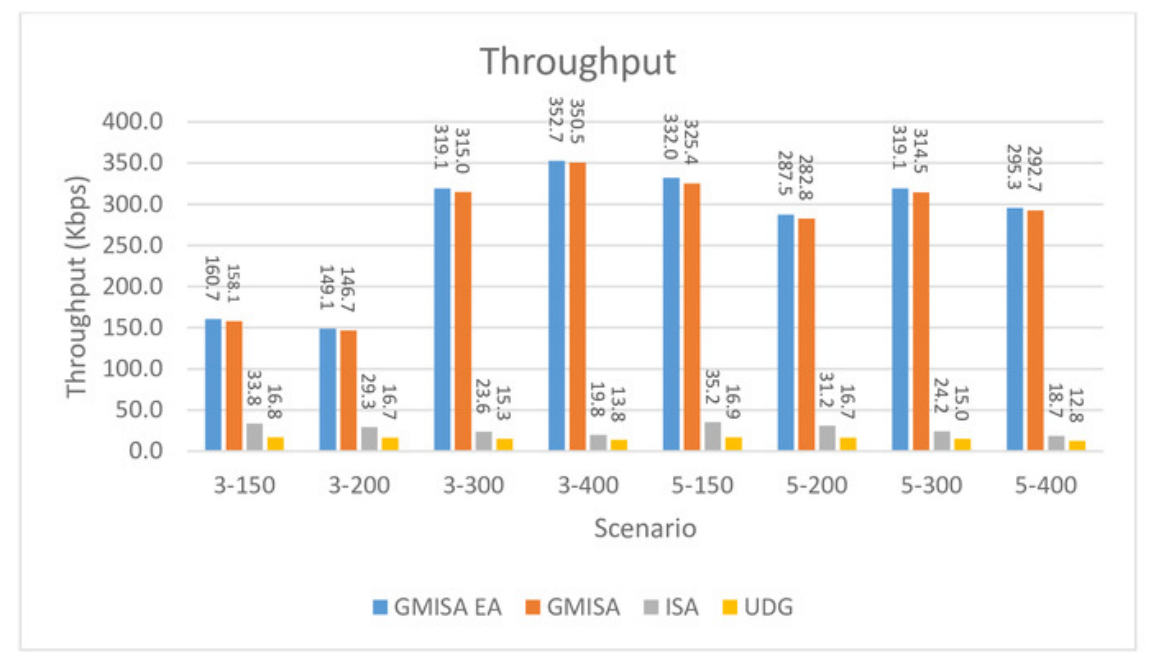




\section{Table $\mathbf{1}$ (on next page)}

Example values for group priority and nodes numbers 
1

2

3

\begin{tabular}{|c|c|c|c|c|}
\hline $\begin{array}{c}\text { Cluster } \\
\text { Number }\end{array}$ & Group $_{\text {priorityLevel }}$ & $\boldsymbol{L}_{\boldsymbol{j}}$ & $\begin{array}{c}\text { Nodes Inside } \\
\text { Group }\end{array}$ & \multirow{2}{*}{$\begin{array}{c}\text { Frame } \\
\text { Size }\end{array}$} \\
\hline \multirow{3}{*}{ C1 } & G1 & 6 & 3 & \multirow{2}{*}{58} \\
\cline { 2 - 4 } & G2 & 5 & 4 & \\
\cline { 2 - 4 } & G3 & 4 & 5 & \multirow{2}{*}{39} \\
\hline \multirow{3}{*}{ C2 } & G2 & 5 & 1 & \multirow{2}{*}{33} \\
\cline { 2 - 4 } & G3 & 4 & 7 & \multirow{2}{*}{19} \\
\cline { 2 - 4 } C3 & G4 & 3 & 2 & \\
\cline { 2 - 4 } & G1 & 6 & 3 & 3 \\
\hline \multirow{3}{*}{ C4 4} & G4 & 3 & 4 & \\
\cline { 2 - 4 } & G5 & 2 & 1 & 5 \\
\hline
\end{tabular}




\section{Table 2 (on next page)}

Simulation Parameters That Are Used For Evaluation 


\begin{tabular}{|l|c|}
\hline \multicolumn{1}{|c|}{ Parameter } & Value \\
\hline Max Packet Life Time (Max PLT) & $30 \mathrm{sec}$ \\
\hline Inter Arrival Time interval (T) & $5-0.25 \mathrm{sec}$ \\
\hline Alert Priority Levels & 16 \\
\hline Number of priority groups (Ng) & $L_{i}=1,2, \ldots 10$ \\
\hline Number of Packet Priority Levels & 16 \\
\hline Superframe Duration (SD) & $0.25 \mathrm{sec}$ \\
\hline Back-off symbol duration & $0.01 \mathrm{sec}$ \\
\hline CCA duration & $0.000128 \mathrm{sec}$ \\
\hline ACK packet size & $18 \mathrm{Bytes}$ \\
\hline Packet Size & $127 \mathrm{Bytes}$ \\
\hline Data Rate & $250 \mathrm{kbps}$ \\
\hline Simulation time & $50 \mathrm{~s}$ \\
\hline macMinBE & 3 \\
\hline macMaxBE & 5 \\
\hline Timeslot Duration (TD) & $0.01 \mathrm{~s}$ \\
\hline Fading type & Nakagami \\
\hline
\end{tabular}




\section{Table 3(on next page)}

Scenarios for internal evaluation of GMAC 


\begin{tabular}{|c|c|}
\hline Scenario No & Number of Cluster-Number of Nodes \\
\hline 1 & $3-150$ \\
\hline 2 & $3-200$ \\
\hline 3 & $5-200$ \\
\hline 4 & $3-300$ \\
\hline 5 & $3-400$ \\
\hline 6 & $5-150$ \\
\hline 7 & $5-300$ \\
\hline 8 & $5-400$ \\
\hline
\end{tabular}

